\title{
Superconducting Magnets for the $12 \mathrm{GeV}$ Upgrade at Jefferson Laboratory
}

\author{
Ruben J. Fair (Senior Member IEEE), 12 GeV Principal Superconducting Magnet Engineer \\ Glenn R. Young, 12 GeV Associate Project Manager, Physics
}

\begin{abstract}
Jefferson Laboratory is embarked on an energy upgrade to its flagship continuous electron beam accelerator in order to expand the scope of its research capabilities and probe further into the structure of nuclear particles. The $12 \mathrm{GeV}$ upgrade includes the design, manufacture, integration, installation and commissioning of eight different superconducting magnets in three separate experimental halls. The effort involves other national laboratories, universities and industry spanning three countries. This paper will summarize the key characteristics of these magnets, ranging in size from 0.2 to $23 \mathrm{MJ}$ in stored energy, and featuring many different types and configurations. The paper will also give an overview of the specific technical challenges for each magnet, and a status report on magnet manufacture and expected delivery dates. The $12 \mathrm{GeV}$ upgrade at $\mathrm{J}$-Lab represents the largest superconducting magnet fabrication and installation program currently ongoing in the United States and this paper will present the breadth of collaborations supporting it.
\end{abstract}

Index Terms-12 GeV upgrade, continuous electron beam accelerator, superconducting magnets, Jefferson Lab

\section{INTRODUCTION}

$\mathrm{W}$ e are at the dawn of a new era in the study of hadronic nuclear physics. The non-Abelian nature of Quantum Chromodynamics (QCD) and the resulting strong coupling at low energies represent a significant challenge to nuclear and particle physicists. The last decade has seen the development of new theoretical and experimental tools to quantitatively study the nature of confinement and the structure of hadrons comprised of light quarks and gluons. Together these will allow both the spectrum and the structure of hadrons to be explained in unprecedented detail. Thus the $12 \mathrm{GeV}$ upgrade of the Continuous Electron Beam Accelerator Facility (CEBAF) at Jefferson Lab (JLab), Newport News, Virginia will enable a new experimental program with substantial discovery potential to address these and other important topics in nuclear, hadronic and electroweak physics.

The upgrade at JLab is presently underway with completion expected in FY16, and will build upon the lab's highly successful superconducting radiofrequency (SRF) acceleration technology. In two of the existing halls new spectrometers are being added, a large acceptance-device called CLAS12 in Hall

Manuscript received August 08, 2014. Authored by Jefferson Science Associates, LLC under U.S. DOE Contract No. DE-AC05-06OR23177. The U.S. Government retains a non-exclusive, paid-up, irrevocable, world-wide license to publish or reproduce this manuscript for U.S. Government purposes.

R. J. Fair and G. R. Young are with the Thomas Jefferson National Accelerator Facility, Newport News, VA 23606 USA, operated by the Jefferson Science Associates for the U.S. Department of Energy Office of Science. Phone: 757-269-6113; fax: 757-269-5279; e-mail: rfair@jlab.org.
$\mathrm{B}$ and a precision magnetic spectrometer called the Super High Momentum Spectrometer, or SHMS, in Hall C. The new experimental Hall D will make use of a tagged bremsstrahlung photon beam and solenoidal detector to house the GlueX experiment. Upgrades to the beam line and associated beam polarimetry in Hall A provide flexibility to include novel oneof-a-kind large-installation experiments. The Central Helium Liquefier at JLab has been doubled in capacity in preparation for this upgrade [1].

This paper will focus on the 8 superconducting magnets ( 7 new and 1 refurbished) planned for installation in Halls B, C and $\mathrm{D}$. The paper will also give an overview of the specific technical challenges for each magnet, and a status report on magnet manufacture and expected delivery dates.

\section{SUPERCONDUCTING MAGNETS}

\section{A. Overview of magnets}

Eight customized superconducting magnets are being built for the physics part of the $12 \mathrm{GeV}$ Upgrade project, accounting for about $\$ 17.1 \mathrm{M}$ out of a project total of $\$ 44.4 \mathrm{M}$. Six of the eight magnets are performance-based subcontracts with external vendors while the remaining two magnets include a refurbished solenoid and a 6-coil torus designed by JLab and the Fermi National Accelerator Laboratory (FNAL). The Cryogenic Control Reservoirs (CCRs), protection and control systems, installation and commissioning for all the magnets are JLab's responsibility. The seven new magnets span a wide range of geometries, magnetic fields and stored energies, not to mention being designed and built by five different vendors in three different countries - which poses its own unique challenges in terms of cost and schedule management as well as technical oversight.

JLab has employed a rigorous Failure Modes and Effects Analysis (FMEA) process to identify areas that require focused technical effort [2]. The timely progression of these tasks was achieved by the formation of a Magnet Task Force to pool critical resources and skills at the laboratory.

The coils for the seven new magnets utilize surplus Superconducting Super Collider (SSC) outer dipole conductor consisting of 36 strands of $0.6 \mathrm{~mm}$ diameter multi-filamentary $\mathrm{NbTi}$ superconductor with a $\mathrm{Cu}: \mathrm{Sc}$ ratio of 1.8:1, manufactured as a flattened Rutherford cable soldered into a nominally dimensioned $2.5 \mathrm{~mm}$ x $20 \mathrm{~mm}$ OFHC copper channel. The superconductor for the magnets has a tested short sample performance of $11000 \mathrm{~A}$ at $4.2 \mathrm{~K}$ and at $5 \mathrm{~T}$. The Hall D solenoid uses a custom design of NbTi cable with 87 large diameter filaments manufactured in the 1970s.

The magnets for Hall B comprise a 6-coil torus and an 
actively shielded $5 \mathrm{~T}$ large bore solenoid both of which are helium conduction cooled. The helium bath cooled magnets for Hall C comprise a Horizontal Bend (HB), a Q1 quadrupole, two additional quadrupoles (Q2 and Q3) and one dipole. The magnet for Hall $\mathrm{D}$ is a refurbished helium bathcooled large bore solenoid from the SLAC National Accelerator Facility (formerly LASS - Large Aperture Superconducting Solenoid, c1972).

All the magnets will use American Magnetics vapor-cooled current leads and will be actively powered using Danfysik water-cooled power supplies. The quench protection system for each magnet consists of an air-cooled dump resistor connected either in series or in parallel with the coils, which is switched into circuit when a quench or other anomaly is detected [3]. Allen-Bradley PLCs will be used for overall magnet and cryogenic control with additional fast data acquisition to capture fault events.

Table I summarizes the key parameters for each of the eight superconducting magnets.

\section{TECHNICAL CHALLENGES AND RISK MITIGATIONS}

One of the hurdles that faced the early stages of the project was to identify a facility that could solder the superconducting cable into copper channels. JLab successfully located a 'start up' company and established strict quality control checks that included an almost continuous presence by JLab engineers at the vendor's site during the entire soldering process.

TABLE I

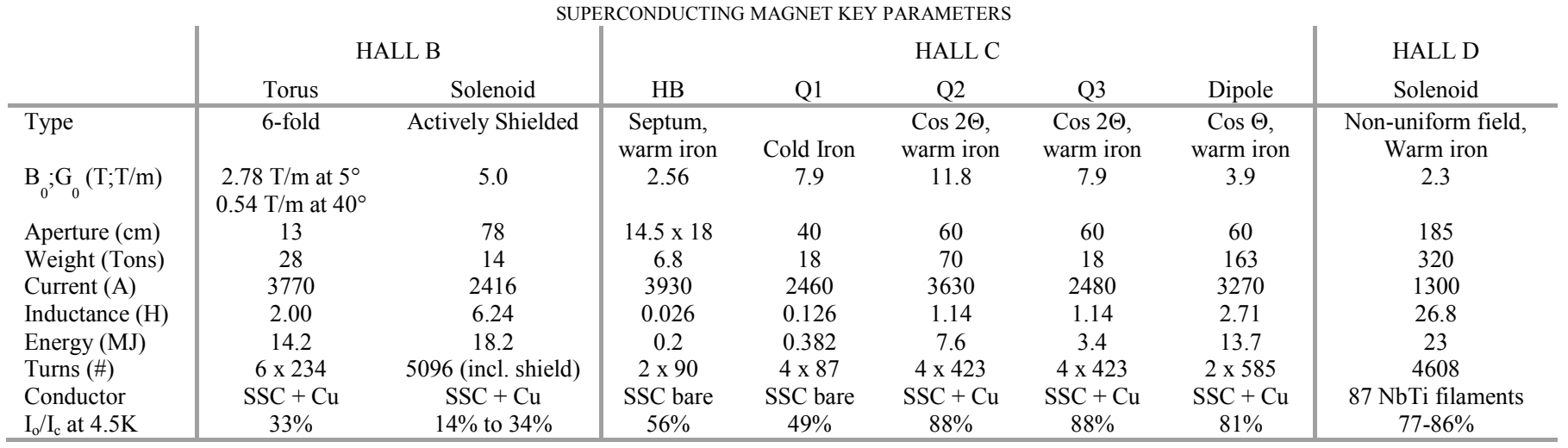

The Hall B torus comprises 6 coils, (each in the shape of a trapezoidal double pancake racetrack using SSC cable soldered into a copper channel), which are being wound and epoxy impregnated by FNAL, and will be electrically connected in series in the final system. Each coil is conduction cooled via a helium cooling tube located along the inside edge of the coil. Thin copper sheets soldered to the cooling tube enclose the entire coil pack. Each coil undergoes two epoxy potting cycles - one in a separate potting mold and the second after the coil is fitted into its aluminum coil case.

A 'Cryostat Factory' will facilitate the assembly of each coil at JLab as it arrives from FNAL. Each coil will be instrumented with temperature sensors and strain gauges and will undergo a cool down test to $80 \mathrm{~K}$ to assess the robustness of the coil's electrical insulation and its structural integrity as well as to test the efficacy of the employed conduction cooling method. The Coil Cold Mass (CCM) will then be wrapped in multi-layer insulation (MLI), fitted with its nitrogen-cooled thermal shield and vacuum jacket before being moved to the experimental hall for final system assembly.

Following the $80 \mathrm{~K}$ test [4] on a full sized $(3.5 \mathrm{~m} \times 2.5 \mathrm{~m})$ practice coil and a subsequent dissection, the coil was discovered to have several 'dry' areas where the epoxy had not penetrated fully. A team was formed to investigate and address this problem and included experts from the USA and overseas organizations. Over a four month period, this team reviewed the impregnation process and carried out multiple trial runs on coil samples with the result that the improved process has now been implemented successfully and coil manufacturing is continuing at FNAL.

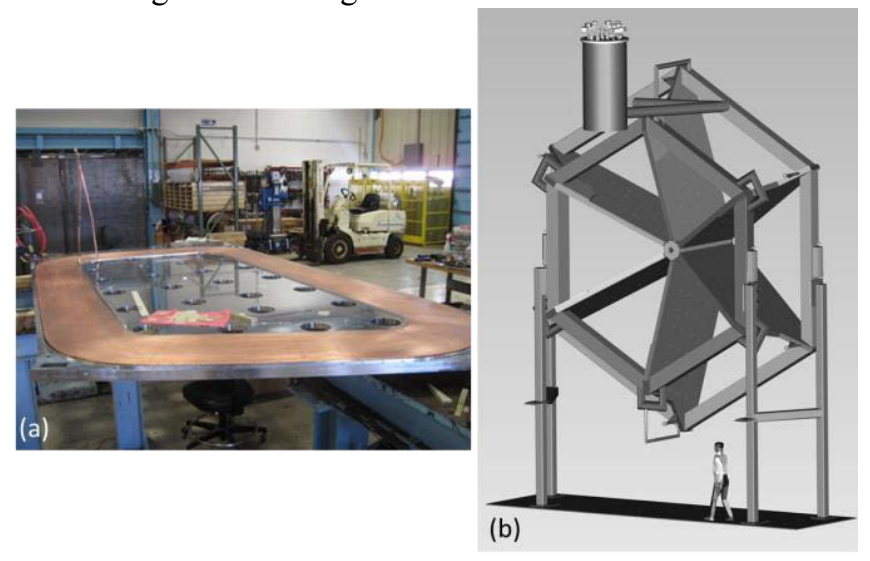

Fig. 1. (a) Torus coil (with copper cooling sheets attached) positioned within its aluminum coil case. (b) CAD model of fully assembled torus

The Hall B solenoid is an actively shielded 5 Tesla magnet consisting of 5 coils using SSC cable soldered into a copper channel and is being designed and built by Everson Tesla Inc. Pennsylvania, USA. Within the hall the solenoid will be located in close proximity to the torus, and both magnets will be completely surrounded by detectors. This physics requirement has also restricted the space available for the magnet coil designs.

The magnet is conduction cooled via liquid helium flowing through a centrally located circumferential channel with copper 'fingers' extending out from the channel and wrapped 
actively shielded $5 \mathrm{~T}$ large bore solenoid both of which are helium conduction cooled. The helium bath cooled magnets for Hall C comprise a Horizontal Bend (HB), a Q1 quadrupole, two additional quadrupoles (Q2 and Q3) and one dipole. The magnet for Hall $\mathrm{D}$ is a refurbished helium bathcooled large bore solenoid from the SLAC National Accelerator Facility (formerly LASS - Large Aperture Superconducting Solenoid, c1972).

All the magnets will use American Magnetics vapor-cooled current leads and will be actively powered using Danfysik water-cooled power supplies. The quench protection system for each magnet consists of an air-cooled dump resistor connected either in series or in parallel with the coils, which is switched into circuit when a quench or other anomaly is detected [3]. Allen-Bradley PLCs will be used for overall magnet and cryogenic control with additional fast data acquisition to capture fault events.

Table I summarizes the key parameters for each of the eight superconducting magnets.

\section{TECHNICAL CHALLENGES AND RISK MITIGATIONS}

One of the hurdles that faced the early stages of the project was to identify a facility that could solder the superconducting cable into copper channels. JLab successfully located a 'start up' company and established strict quality control checks that included an almost continuous presence by JLab engineers at the vendor's site during the entire soldering process.

TABLE I

\begin{tabular}{|c|c|c|c|c|c|c|c|c|}
\hline \multicolumn{9}{|c|}{ SUPERCONDUCTING MAGNET KEY PARAMETERS } \\
\hline Type & 6-fold & Actively Shielded & $\begin{array}{l}\text { Septum, } \\
\text { warm iron }\end{array}$ & Cold Iron & $\begin{array}{l}\text { Cos } 2 \Theta, \\
\text { warm iron }\end{array}$ & $\begin{array}{c}\text { Cos } 2 \Theta, \\
\text { warm iron }\end{array}$ & $\begin{array}{c}\text { Cos } \Theta, \\
\text { warm iron }\end{array}$ & $\begin{array}{l}\text { Non-uniform field, } \\
\text { Warm iron }\end{array}$ \\
\hline $\mathrm{B}_{0} ; \mathrm{G}_{0}(\mathrm{~T} ; \mathrm{T} / \mathrm{m})$ & $\begin{array}{c}2.78 \mathrm{~T} / \mathrm{m} \text { at } 5^{\circ} \\
0.54 \mathrm{~T} / \mathrm{m} \text { at } 40^{\circ}\end{array}$ & 5.0 & 2.56 & 7.9 & 11.8 & 7.9 & 3.9 & 2.3 \\
\hline Aperture (cm) & 13 & 78 & $14.5 \times 18$ & 40 & 60 & 60 & 60 & 185 \\
\hline Weight (Tons) & 28 & 14 & 6.8 & 18 & 70 & 18 & 163 & 320 \\
\hline Energy (MJ) & 14.2 & 18.2 & 0.2 & 0.382 & 7.6 & 3.4 & 13.7 & 23 \\
\hline Turns (\#) & $6 \times 234$ & 5096 (incl. shield) & $2 \times 90$ & $4 \times 87$ & $4 \times 423$ & $4 \times 423$ & $2 \times 585$ & 4608 \\
\hline Conductor & $\mathrm{SSC}+\mathrm{Cu}$ & $\mathrm{SSC}+\mathrm{Cu}$ & SSC bare & SSC bare & $\mathrm{SSC}+\mathrm{Cu}$ & $\mathrm{SSC}+\mathrm{Cu}$ & $\mathrm{SSC}+\mathrm{Cu}$ & $87 \mathrm{NbTi}$ filaments \\
\hline $\mathrm{I}_{0} / \mathrm{I}_{\mathrm{c}}$ at $4.5 \mathrm{~K}$ & $33 \%$ & $14 \%$ to $34 \%$ & $56 \%$ & $49 \%$ & $88 \%$ & $88 \%$ & $81 \%$ & $77-86 \%$ \\
\hline
\end{tabular}

The Hall B torus comprises 6 coils, (each in the shape of a trapezoidal double pancake racetrack using SSC cable soldered into a copper channel), which are being wound and epoxy impregnated by FNAL, and will be electrically connected in series in the final system. Each coil is conduction cooled via a helium cooling tube located along the inside edge of the coil. Thin copper sheets soldered to the cooling tube enclose the entire coil pack. Each coil undergoes two epoxy potting cycles - one in a separate potting mold and the second after the coil is fitted into its aluminum coil case.

A 'Cryostat Factory' will facilitate the assembly of each coil at JLab as it arrives from FNAL. Each coil will be instrumented with temperature sensors and strain gauges and will undergo a cool down test to $80 \mathrm{~K}$ to assess the robustness of the coil's electrical insulation and its structural integrity as well as to test the efficacy of the employed conduction cooling method. The Coil Cold Mass (CCM) will then be wrapped in multi-layer insulation (MLI), fitted with its nitrogen-cooled thermal shield and vacuum jacket before being moved to the experimental hall for final system assembly.

Following the $80 \mathrm{~K}$ test [4] on a full sized $(3.5 \mathrm{~m} \times 2.5 \mathrm{~m})$ practice coil and a subsequent dissection, the coil was discovered to have several 'dry' areas where the epoxy had not penetrated fully. A team was formed to investigate and address this problem and included experts from the USA and overseas organizations. Over a four month period, this team reviewed the impregnation process and carried out multiple trial runs on coil samples with the result that the improved process has now been implemented successfully and coil manufacturing is continuing at FNAL.

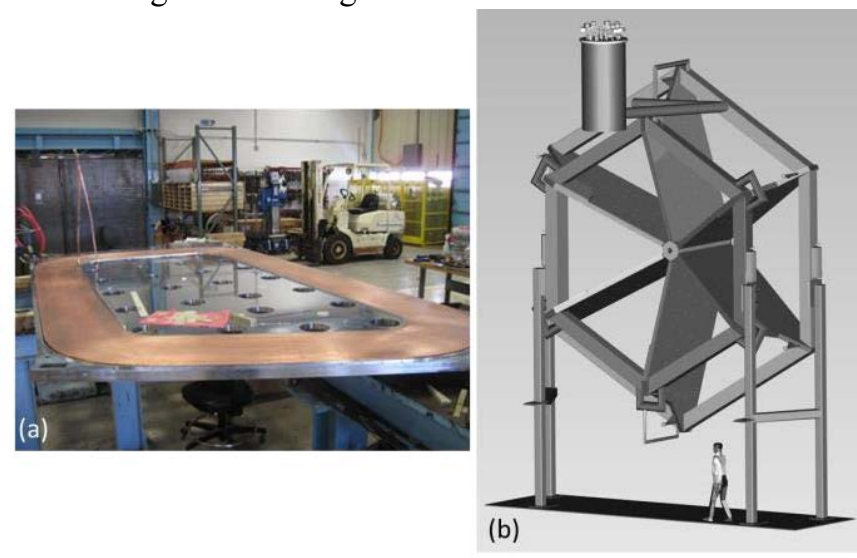

Fig. 1. (a) Torus coil (with copper cooling sheets attached) positioned within its aluminum coil case. (b) CAD model of fully assembled torus

The Hall B solenoid is an actively shielded 5 Tesla magnet consisting of 5 coils using SSC cable soldered into a copper channel and is being designed and built by Everson Tesla Inc. Pennsylvania, USA. Within the hall the solenoid will be located in close proximity to the torus, and both magnets will be completely surrounded by detectors. This physics requirement has also restricted the space available for the magnet coil designs.

The magnet is conduction cooled via liquid helium flowing through a centrally located circumferential channel with copper 'fingers' extending out from the channel and wrapped 
round the individual coils, the entire assembly then being potted in epoxy. Helium gas exiting from the coil cooling channel is used to cool the thermal shield before being exhausted via the CCR.

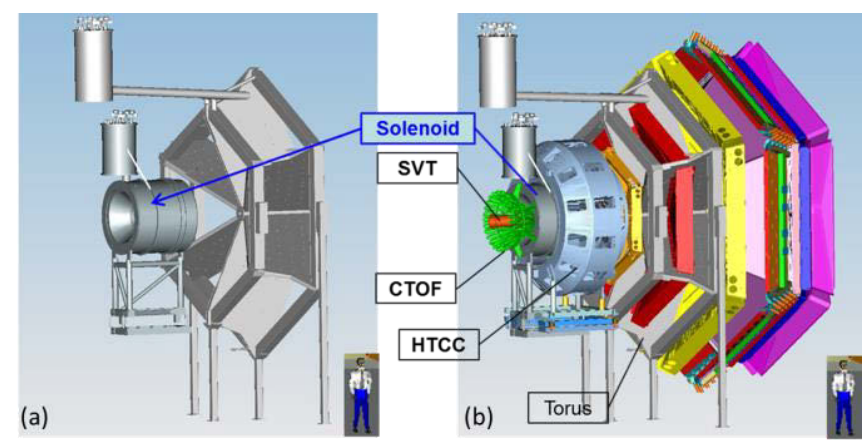

Fig. 2. (a) Location of the torus with respect to the Solenoid, (b) Detectors surrounding both magnets.

Studies indicated that the design is sensitive to manufacturing variations which could degrade the magnetic field homogeneity from its specified value of $<100 \mathrm{ppm}$ (peakto-peak) within a cylindrical volume of $25 \mathrm{~mm}$ diameter $\mathrm{x} 40$ $\mathrm{mm}$ length located at the geometric center of the magnet. A proposed solution is to incorporate small superconducting shims (Z1, Z2, $\mathrm{X}$ and $\mathrm{Y})$ on the $1 \mathrm{~K}$ shield which surrounds the target within the bore of the magnet. In order to quantify manufacturing variations for the solenoid coils and to check on the effectiveness of the epoxy impregnation process, a halfsize practice coil will be wound, potted and dissected.

A potential failure with coils 'sticking and slipping' against their formers during current ramp-up, thereby causing spurious quenching, necessitated the design of slip planes to overcome this problem. Forces and stresses encountered within the thermal radiation shield during quench events have been mitigated by slotting the shield. Thermal differential stresses during cool down have been minimized. Thermal margins for each coil have been quantified resulting in improvements to the design and operation of the overall cryogenic cooling scheme. Electromagnetic interactions between the torus and the solenoid were studied confirming that only under certain fault conditions were the effects significant but also that they could be mitigated, both at the design and manufacturing stages and via the planned control and protection systems [5].

The Hall C Horizontal Bend (HB) dipole magnet is a compact, high current density "C" type superferric dipole magnet which is helium bath cooled possessing a warm iron yoke and is being built by the National Superconducting Cyclotron Lab at Michigan State University (NSCL/MSU).

The major challenges here are the tight geometry, high and unbalanced forces and a required low fringe field in the primary beam path. The coil design is based on Kapton insulated flattened SSC Rutherford cable (wound in a traditional saddle configuration) that provides a large current margin, and commercially available fiberglass pre-preg epoxy tape. The superconducting cable has been re-rolled to remove the $0.9^{\circ}$ keystone angle with only an approximate $1 \%$ loss of short sample performance after re-rolling [6].

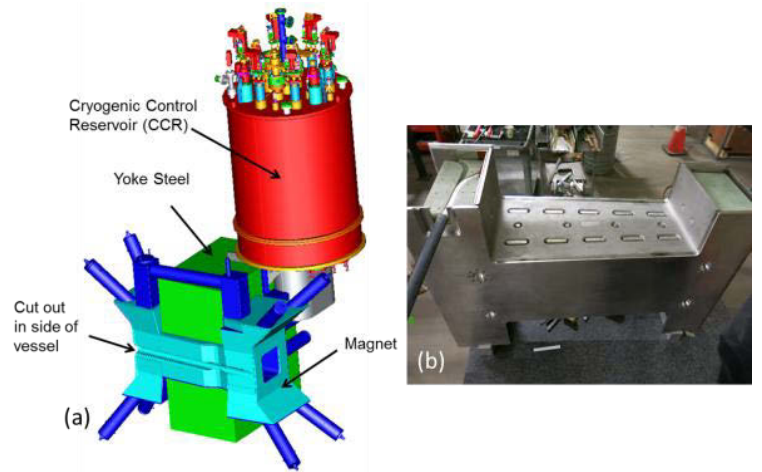

Fig. 3. (a) The HB magnet showing the cut out in the side of the vessel to accommodate the beam line for the HMS (b) Welded helium vessel

The installation of the coils within the helium vessel and the subsequent vessel closure welds were carefully controlled processes utilizing a multi-step checking procedure to minimize distortions due to welding. The thermal radiation shield and vacuum vessel are then built around the MLIwrapped helium vessel. Radial clearances within the magnet are extremely tight and there is a risk of small thermal shorts being present even after the final assembly. Due to the possibility of high radiation dose levels within Hall C, where possible and available, radiation-tolerant materials have been used for the construction of the magnet. Studies are ongoing at JLab to quantify the effects of radiation dose on the structural integrity of the various components of the magnet.

The magnet will undergo a room temperature plot at MSU and will have its CCR fitted before being shipped to JLab where its warm iron yoke will be assembled within the hall.

The Hall C Q1 quadrupole magnet is being designed and built by Scientific Magnetics in England, uses bare Rutherford cable for its superconducting coils and is helium bath cooled.
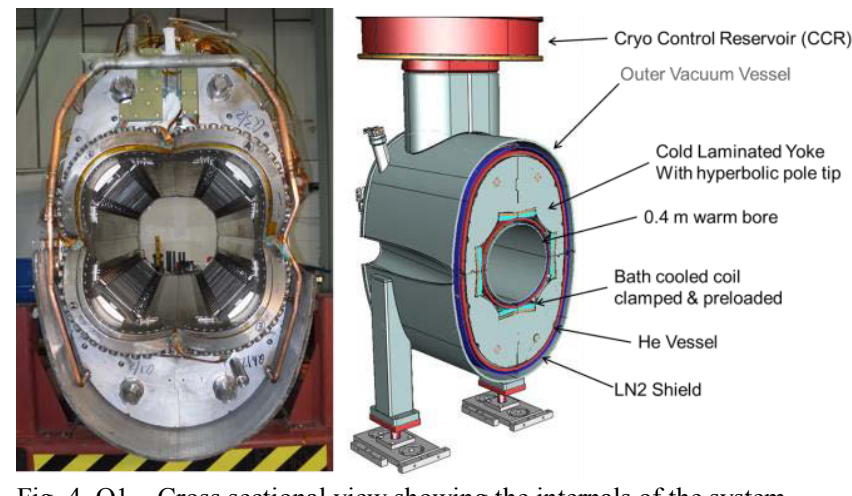

Fig. 4. Q1 - Cross sectional view showing the internals of the system

The four coils are clamped and preloaded within a cold laminated iron yoke. The helium vessel and nitrogen shield are wrapped with MLI before the outer vacuum jacket is finally fitted and welded. The magnet has had its magnetic field profile successfully validated at room temperature. The CCR will be fitted by the vendor before shipping. The key challenges here have been the fabrication of large vessels ( 2.4 $\mathrm{m}$ long $\times 1.5 \mathrm{~m}$ diameter) to close tolerances and the control and verification of welding processes and materials used to 
meet required standards. As with any bespoke magnet build, lessons were learned and solutions were implemented as the build progressed [7].

The Hall C Q2 and Q3 superconducting cosine $2 \Theta$ quadrupoles are being built by Sigma Phi in France and are designed as bath-cooled magnets with coils that are supported mechanically using segmented aluminum force collars. The Q2 and Q3 quad coils consist of four double layers of SSC cable soldered into a copper channel. These magnets are cryogenically identical but have different warm iron yokes optimized for each magnets actual excitation. The requirements of the SHMS spectrometer require that the Q2 quad operates at or near $(83 \%)$ its design maximum gradient, while the Q3 quad only operates at $60 \%$ of its required excitation thus affording some measure of redundancy if the final performances of the Q2 and Q3 magnets turn out not to be equal [8].

At the time of writing, manufacture has not commenced for these magnets with the dipole being worked on first.
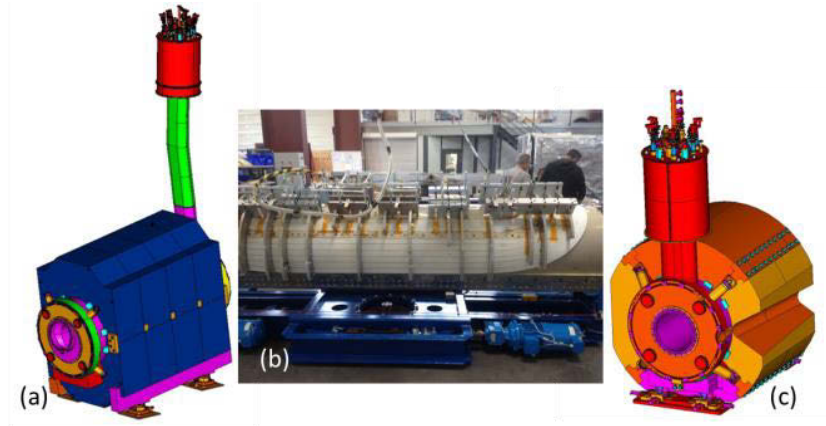

Fig. 5. (a) $\operatorname{Cos} \Theta$ Dipole (b) Production dipole coil (c) Q2 Cos $2 \Theta$ quadrupole

The Hall C Dipole magnet is helium bath cooled and is a two sector cosine $\Theta$ dipole also being manufactured by Sigma Phi. It is wound with three double layers of SSC cable soldered into a copper channel and also features a segmented shrink fit force collar to clamp the coils [9]. The need for a segmented force collar was determined after transient studies of a fast discharge of the magnet indicated that nearly $2 \mathrm{MJ}$ of heat energy was being deposited in the force collar whereas there was only $1 \mathrm{MJ}$ of latent heat of helium in the dipole cryostat [10].

The conductor turned out to be too 'soft' with a yield strength close to expected compressive loads. The magnet subcontractor successfully introduced a conductor consolidation process which hardened the conductor to provide a $50 \%$ safety margin at $4 \mathrm{~K}$ [11].

The Hall D Solenoid, built at SLAC in the 1970s, and refurbished at JLab, consists of four superconducting coils in four separate helium baths, and is about $4 \mathrm{~m}$ long with a $1.8 \mathrm{~m}$ diameter bore and has a stored energy of approximately $23 \mathrm{MJ}$ at $1300 \mathrm{~A}$. It was first cooled down and powered in May 2013 and successfully ramped to $1500 \mathrm{~A}$. A subsequent ramp caused the magnet to quench at $1460 \mathrm{~A}$. The magnet was then run multiple times to 1350 A without mishap [12]. A detailed investigation was conducted as to the cause of the quench but a root cause could not be determined.

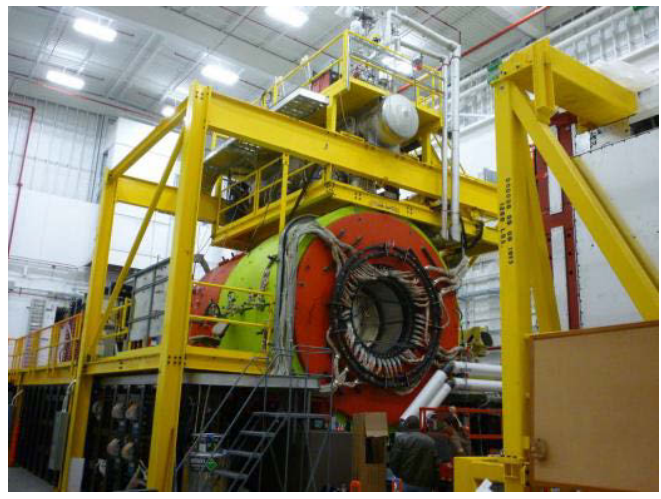

Fig. 6. Hall D solenoid

TABLE 2

HALL D SOLENOID QUENCH INVESTIGATION RESULTS

\begin{tabular}{ll}
\hline \hline Possible Fault & Evaluation as Cause \\
\hline Problems at splices & Very unlikely \\
Damaged conductor & Very unlikely \\
Higher temperature at time of quench & Unlikely \\
Gas bubble exposed conductor & Unlikely \\
Flux jumping & Possible \\
Conductor motion & Possible \\
Short & No \\
\hline
\end{tabular}

Examination of the planned physics program indicated that 1300 A was a safe operating current for this solenoid.

\section{CURRENT STATUS AND EXPECTED DELIVERY DATES}

At the time of writing, the status and expected delivery dates for each magnet is as summarized in Table 3 below.

TABLE 3

STATUS AND EXPECTED DELIVERY DATES

\begin{tabular}{lll}
\hline \hline \multicolumn{1}{c}{ Magnet } & Status & $\begin{array}{c}\text { Expected Magnet } \\
\text { Delivery Date }\end{array}$ \\
\hline Hall B Torus & Coil \#1 being manufactured & Quarter 4 FY15 \\
Hall B Solenoid & Practice Coil being manufactured & Quarter 2 FY16 \\
Hall C HB & Vacuum vessel being assembled & Quarter 4 FY14 \\
Hall C Q1 & Closure welding on OVC & Quarter 3 FY14 \\
Hall C Q2 & Conductor consolidation & Quarter 3 FY15 \\
Hall C Q3 & Conductor consolidation & Quarter 1 FY16 \\
Hall C Dipole & Second coil nearing completion & Quarter 3 FY15 \\
Hall D Solenoid & Cold at 4.5K & Ready to run \\
\hline
\end{tabular}

\section{CONCLUSION}

The formation of the Magnet Task Force and the sharing of 'lessons learned' between the experimental halls and the various superconducting magnet designs is already showing great promise for a successful outcome for the $12 \mathrm{GeV}$ upgrade project. The capabilities of the upgraded CEBAF will represent a significant leap over previous technology, with an unmatched combination of beam energy, quality and intensity which provides innovative opportunities for probing (and extending) the Standard Model. Jefferson Lab is providing the US nuclear physics program with a leadership role in this vibrant international community studying QCD, confinement, and precision tests of the Standard Model, and with the successful upgrade, will be a flagship for this diverse scientific program for many years to come. 


\section{ACKNOWLEDGMENT}

This work was supported in part by the U.S. Department of Energy under U.S. DOE Contract No. DE-AC05-06OR23177. The authors would like to acknowledge the contributions and support of the Magnet Task Force team members, technicians, support staff and management at JLab. We would also like to thank all the vendors FNAL, NSCL/MSU, Everson Tesla, Sigma Phi, Scientific Magnetics and SLAC for their valued insights and shared experiences in designing and building these technically challenging magnet systems.

\section{REFERENCES}

[1] J. Dudek, R. Ent, R. Essig, K. Kumar, C. Meyer, R. McKeown, Z. E. Meziani, G. A. Miller, M. Pennington, D. Richards, L. Weinstein, G. Young and S. Brown, "Physics opportunities with the $12 \mathrm{GeV}$ Upgrade at Jefferson Lab," U.S. Department of Energy, Jefferson Science Associates, August 2012.

[2] P K Ghoshal, R J Fair, R Rajput-Ghoshal, G Biallas, W Schneider, R Legg, D Kashy, J Hogan, M Wiseman, G Young, L. Elouadhiri, C Rode, "FMEA on the superconducting Torus for the Jefferson Lab $12 \mathrm{GeV}$ accelerator upgrade", IEEE Trans. on Appl. Supercond., ASC 2014

[3] O. Pastor, T. Willard, P. K. Ghoshal, D. Kashy, M. Wiseman, V. S. Kashikhin, G. Young, L. Elouadhiri, C. Rode, "Eddy current and quench analysis on thermal shield in Torus magnet for $12 \mathrm{GeV}$ upgrade", IEEE Trans. on Appl. Supercond., ASC 2014

[4] Robert Legg, David Kashy, Ruben Fair, Probir Ghoshal, Rama Bachimanchi, Krister Bruhwel, , Danny Machie, Jacob Powers, "Liquid nitrogen tests of a Torus coil for the Jefferson Lab $12 \mathrm{GeV}$ accelerator upgrade", IEEE Trans. on Appl. Supercond., ASC 2014
[5] R. Rajput-Ghoshal, R. J. Fair, P. K. Ghoshal, J. Hogan, D. Kashy, V. Burkert, "An investigation into the electromagnetic interactions between a superconducting Torus and solenoid for the Jefferson Lab $12 \mathrm{GeV}$ accelerator upgrade", IEEE Trans. on Appl. Supercond., ASC 2014

[6] S. Chouhan, J. DeKamp, E. E. Burkhardt, H. Song, M. Leitner, A. Zeller, P. D. Brindza, E. Sun, S. R. Lassiter, M. Fowler, "Design and Fabrication of the Superconducting Horizontal Bend Magnet for the Super High Momentum Spectrometer at JLab", IEEE Trans. on Appl. Supercond., ASC 2014

[7] S. R. Lassiter, P. D. Brindza, E. Sun, M. Fowler, S. R. Milward, F. P. Domptail, P. N. Penfold, "Construction and Testing of JLAB's Cold Iron Superconducting Q1 Magnet for the Super High Momentum Spectrometer", IEEE Trans. on Appl. Supercond., ASC 2014

[8] P. D. Brindza, S. Lassiter, M. Fowler, E. Sun, B. Metzger, P. Medeiros, D. Young, S. Furches, "Design of the Superconducting Super High Momentum Spectrometer (SHMS) for the JLAB $12 \mathrm{GeV}$ Upgrade," IEEE Trans. Appl. Supercond., Chicago, August 2008.

[9] S. Lassiter et al, "Structural Analysis of the SHMS Cosine Theta Superconducting Dipole Force Collar", IEEE Trans. on Appl. Supercond., Vol 19, no 3, June 2009

[10] E. Sun, "Eddy Current Heat Calculation for the Dipole and the Q2/3", Hall C Engineering Note, 2008.

http://hallcweb.jlab.org/doc-public/DocumentDatabase

[11] E. Sun, G. Cheng, S. R. Lassiter, P. D. Brindza, M. Fowler, F. Forest, A. Porhiel, S. Antoine, "Analysis of the Superconducting Dipole Coil of 11 $\mathrm{GeV}$ Super High Momentum Spectrometer", IEEE Trans. on Appl. Supercond., ASC 2014

[12] George Biallas, et. al.,"Commissioning and Testing the 1970's Era LASS Solenoid Magnet in JLab's Hall D”, IEEE Trans. on Appl. Supercond., ASC 2014 
round the individual coils, the entire assembly then being potted in epoxy. Helium gas exiting from the coil cooling channel is used to cool the thermal shield before being exhausted via the CCR.

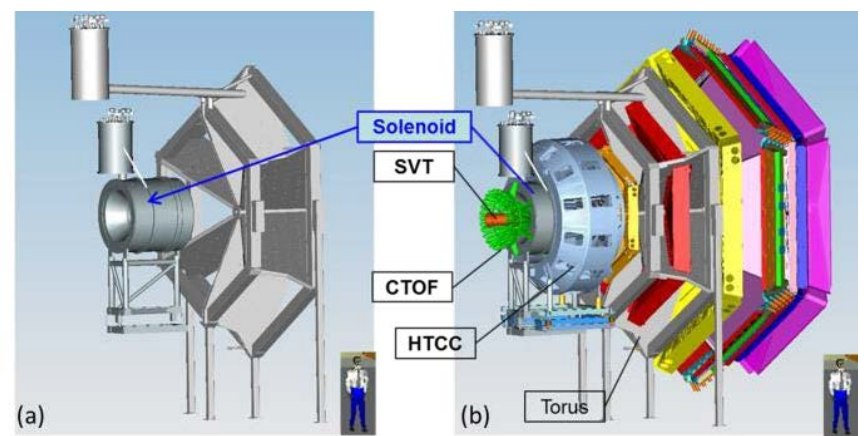

Fig. 2. (a) Location of the torus with respect to the Solenoid, (b) Detectors surrounding both magnets.

Studies indicated that the design is sensitive to manufacturing variations which could degrade the magnetic field homogeneity from its specified value of $<100 \mathrm{ppm}$ (peakto-peak) within a cylindrical volume of $25 \mathrm{~mm}$ diameter $\mathrm{x} 40$ $\mathrm{mm}$ length located at the geometric center of the magnet. A proposed solution is to incorporate small superconducting shims (Z1, Z2, $\mathrm{X}$ and $\mathrm{Y})$ on the $1 \mathrm{~K}$ shield which surrounds the target within the bore of the magnet. In order to quantify manufacturing variations for the solenoid coils and to check on the effectiveness of the epoxy impregnation process, a halfsize practice coil will be wound, potted and dissected.

A potential failure with coils 'sticking and slipping' against their formers during current ramp-up, thereby causing spurious quenching, necessitated the design of slip planes to overcome this problem. Forces and stresses encountered within the thermal radiation shield during quench events have been mitigated by slotting the shield. Thermal differential stresses during cool down have been minimized. Thermal margins for each coil have been quantified resulting in improvements to the design and operation of the overall cryogenic cooling scheme. Electromagnetic interactions between the torus and the solenoid were studied confirming that only under certain fault conditions were the effects significant but also that they could be mitigated, both at the design and manufacturing stages and via the planned control and protection systems [5].

The Hall C Horizontal Bend (HB) dipole magnet is a compact, high current density "C" type superferric dipole magnet which is helium bath cooled possessing a warm iron yoke and is being built by the National Superconducting Cyclotron Lab at Michigan State University (NSCL/MSU).

The major challenges here are the tight geometry, high and unbalanced forces and a required low fringe field in the primary beam path. The coil design is based on Kapton insulated flattened SSC Rutherford cable (wound in a traditional saddle configuration) that provides a large current margin, and commercially available fiberglass pre-preg epoxy tape. The superconducting cable has been re-rolled to remove the $0.9^{\circ}$ keystone angle with only an approximate $1 \%$ loss of short sample performance after re-rolling [6].

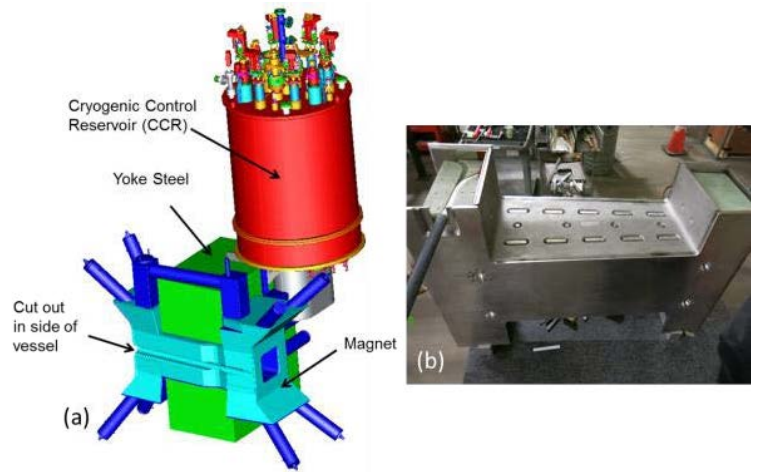

Fig. 3. (a) The HB magnet showing the cut out in the side of the vessel to accommodate the beam line for the HMS (b) Welded helium vessel

The installation of the coils within the helium vessel and the subsequent vessel closure welds were carefully controlled processes utilizing a multi-step checking procedure to minimize distortions due to welding. The thermal radiation shield and vacuum vessel are then built around the MLIwrapped helium vessel. Radial clearances within the magnet are extremely tight and there is a risk of small thermal shorts being present even after the final assembly. Due to the possibility of high radiation dose levels within Hall C, where possible and available, radiation-tolerant materials have been used for the construction of the magnet. Studies are ongoing at JLab to quantify the effects of radiation dose on the structural integrity of the various components of the magnet.

The magnet will undergo a room temperature plot at MSU and will have its CCR fitted before being shipped to JLab where its warm iron yoke will be assembled within the hall.

The Hall C Q1 quadrupole magnet is being designed and built by Scientific Magnetics in England, uses bare Rutherford cable for its superconducting coils and is helium bath cooled.
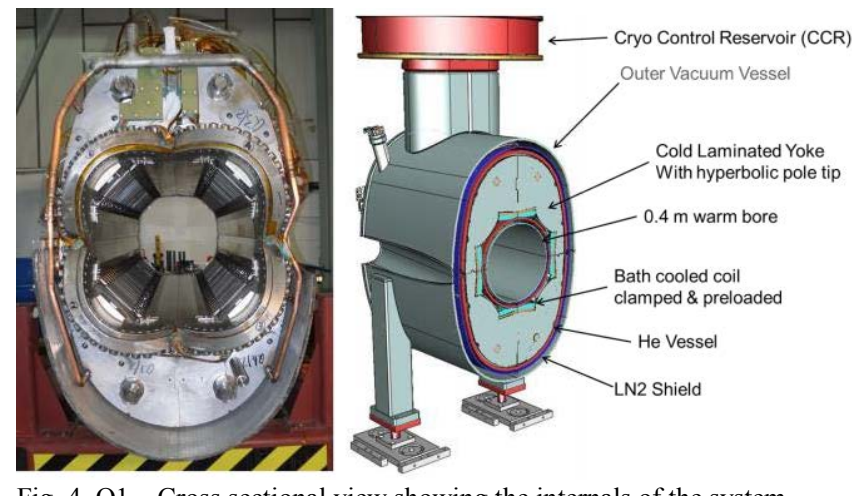

Fig. 4. Q1 - Cross sectional view showing the internals of the system

The four coils are clamped and preloaded within a cold laminated iron yoke. The helium vessel and nitrogen shield are wrapped with MLI before the outer vacuum jacket is finally fitted and welded. The magnet has had its magnetic field profile successfully validated at room temperature. The CCR will be fitted by the vendor before shipping. The key challenges here have been the fabrication of large vessels ( 2.4 $\mathrm{m}$ long $\times 1.5 \mathrm{~m}$ diameter) to close tolerances and the control and verification of welding processes and materials used to 
meet required standards. As with any bespoke magnet build, lessons were learned and solutions were implemented as the build progressed [7].

The Hall C Q2 and Q3 superconducting cosine $2 \Theta$ quadrupoles are being built by Sigma Phi in France and are designed as bath-cooled magnets with coils that are supported mechanically using segmented aluminum force collars. The Q2 and Q3 quad coils consist of four double layers of SSC cable soldered into a copper channel. These magnets are cryogenically identical but have different warm iron yokes optimized for each magnets actual excitation. The requirements of the SHMS spectrometer require that the Q2 quad operates at or near $(83 \%)$ its design maximum gradient, while the Q3 quad only operates at $60 \%$ of its required excitation thus affording some measure of redundancy if the final performances of the Q2 and Q3 magnets turn out not to be equal [8].

At the time of writing, manufacture has not commenced for these magnets with the dipole being worked on first.
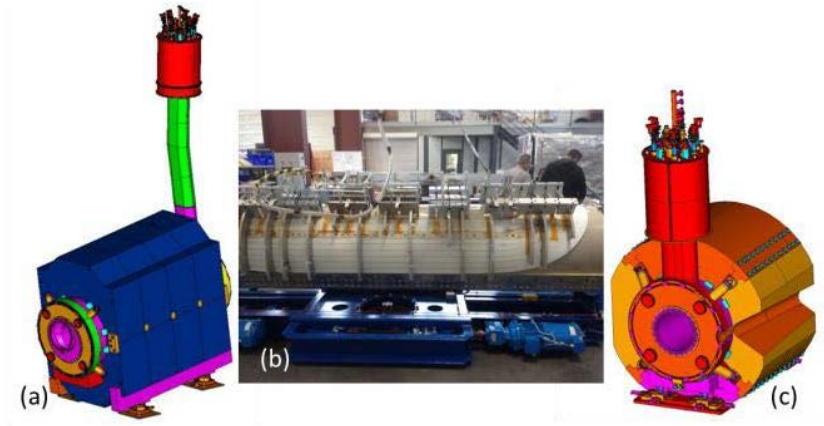

Fig. 5. (a) $\operatorname{Cos} \Theta$ Dipole (b) Production dipole coil (c) Q2 Cos $2 \Theta$ quadrupole

The Hall C Dipole magnet is helium bath cooled and is a two sector cosine $\Theta$ dipole also being manufactured by Sigma Phi. It is wound with three double layers of SSC cable soldered into a copper channel and also features a segmented shrink fit force collar to clamp the coils [9]. The need for a segmented force collar was determined after transient studies of a fast discharge of the magnet indicated that nearly $2 \mathrm{MJ}$ of heat energy was being deposited in the force collar whereas there was only $1 \mathrm{MJ}$ of latent heat of helium in the dipole cryostat [10].

The conductor turned out to be too 'soft' with a yield strength close to expected compressive loads. The magnet subcontractor successfully introduced a conductor consolidation process which hardened the conductor to provide a $50 \%$ safety margin at $4 \mathrm{~K}$ [11].

The Hall D Solenoid, built at SLAC in the 1970s, and refurbished at JLab, consists of four superconducting coils in four separate helium baths, and is about $4 \mathrm{~m}$ long with a $1.8 \mathrm{~m}$ diameter bore and has a stored energy of approximately $23 \mathrm{MJ}$ at $1300 \mathrm{~A}$. It was first cooled down and powered in May 2013 and successfully ramped to $1500 \mathrm{~A}$. A subsequent ramp caused the magnet to quench at $1460 \mathrm{~A}$. The magnet was then run multiple times to 1350 A without mishap [12]. A detailed investigation was conducted as to the cause of the quench but a root cause could not be determined.

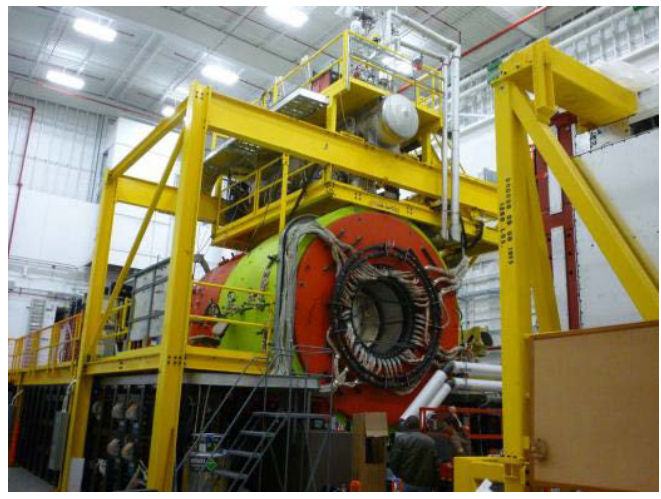

Fig. 6. Hall D solenoid

TABLE 2

HALL D SOLENOID QUENCH INVESTIGATION RESULTS

\begin{tabular}{ll}
\hline \hline Possible Fault & Evaluation as Cause \\
\hline Problems at splices & Very unlikely \\
Damaged conductor & Very unlikely \\
Higher temperature at time of quench & Unlikely \\
Gas bubble exposed conductor & Unlikely \\
Flux jumping & Possible \\
Conductor motion & Possible \\
Short & No \\
\hline
\end{tabular}

Examination of the planned physics program indicated that 1300 A was a safe operating current for this solenoid.

\section{CURRENT STATUS AND EXPECTED DELIVERY DATES}

At the time of writing, the status and expected delivery dates for each magnet is as summarized in Table 3 below.

TABLE 3

STATUS AND EXPECTED DELIVERY DATES

\begin{tabular}{lll}
\hline \hline \multicolumn{1}{c}{ Magnet } & Status & $\begin{array}{c}\text { Expected Magnet } \\
\text { Delivery Date }\end{array}$ \\
\hline Hall B Torus & Coil \#1 being manufactured & Quarter 4 FY15 \\
Hall B Solenoid & Practice Coil being manufactured & Quarter 2 FY16 \\
Hall C HB & Vacuum vessel being assembled & Quarter 4 FY14 \\
Hall C Q1 & Closure welding on OVC & Quarter 3 FY14 \\
Hall C Q2 & Conductor consolidation & Quarter 3 FY15 \\
Hall C Q3 & Conductor consolidation & Quarter 1 FY16 \\
Hall C Dipole & Second coil nearing completion & Quarter 3 FY15 \\
Hall D Solenoid & Cold at 4.5K & Ready to run \\
\hline
\end{tabular}

\section{CONCLUSION}

The formation of the Magnet Task Force and the sharing of 'lessons learned' between the experimental halls and the various superconducting magnet designs is already showing great promise for a successful outcome for the $12 \mathrm{GeV}$ upgrade project. The capabilities of the upgraded CEBAF will represent a significant leap over previous technology, with an unmatched combination of beam energy, quality and intensity which provides innovative opportunities for probing (and extending) the Standard Model. Jefferson Lab is providing the US nuclear physics program with a leadership role in this vibrant international community studying QCD, confinement, and precision tests of the Standard Model, and with the successful upgrade, will be a flagship for this diverse scientific program for many years to come. 